\title{
An online intervention for improving stroke survivors' health-related quality of life: study protocol for a randomised controlled trial
}

Ashleigh Guillaumier ${ }^{1,3}$ D , Sam McCrabb , Neil J. Spratt ${ }^{2,3,4}$, Michael Pollack ${ }^{1,4}$, Amanda L. Baker ${ }^{1,3}$, Parker Magin ${ }^{1}$, Alyna Turner ${ }^{1,5,6}$, Christopher Oldmeadow ${ }^{1,3}$, Clare Collins ${ }^{3,7}$, Robin Callister ${ }^{2,3}$, Chris Levi ${ }^{1}$, Andrew Searles ${ }^{1,3}$, Simon Deeming ${ }^{1,3}$, Olivia Wynne ${ }^{1}$, Alexandra M. J. Denham ${ }^{1,3}$, Brigid Clancy ${ }^{1}$ and Billie Bonevski ${ }^{1,3^{*}}$

\begin{abstract}
Background: Recurrent stroke is a major contributor to stroke-related disability and costs. Improving health-risk behaviours and mental health has the potential to significantly improve recovery, enhance health-related quality of life (HRQoL), independent living, and lower the risk of recurrent stroke. The primary aim will be to test the effectiveness of an online intervention to improve HRQoL among stroke survivors at 6 months' follow-up. Programme effectiveness on four health behaviours, anxiety and depression, cost-effectiveness, and impact on other hospital admissions will also be assessed.

Methods/design: An open-label randomised controlled trial is planned. A total of 530 adults will be recruited across one national and one regional stroke registry and block randomised to the intervention or minimal care control group. The intervention group will receive access to the online programme Prevent 2 nd Stroke (P2S); the minimal care control group will receive an email with Internet addresses of generic health sites designed for the general population. The primary outcome, HRQoL, will be measured using the EuroQol-5D. A full analysis plan will compare between groups from baseline to follow-up.
\end{abstract}

Discussion: A low-cost per user option to supplement current care, such as P2S, has the potential to increase HRQOL for stroke survivors, and reduce the risk of second stroke.

Trial registration: Australian and New Zealand Clinical Trials Registry, ID: ACTRN12617001205325p. Registered on 17 August 2017.

Keywords: Recurrent stroke, Prevention, Health behaviours, Health-related quality of life

\section{Background}

Hypertension, smoking tobacco, low levels of physical activity, poor diet, and heavy levels of alcohol consumption are risk factors for recurrent stroke, [1] and can impact on health-related quality of life (HRQoL) [2-6]. Poor health behaviours can increase the risk of a second

\footnotetext{
* Correspondence: billie.bonevski@newcastle.edu.au

${ }^{1}$ School of Medicine and Public Health, Faculty of Health and Medicine, The University of Newcastle, 1 University Drive, Callaghan, NSW 2308, Australia

${ }^{3}$ Hunter Medical Research Institute (HMRI), New Lambton Heights, NSW 2305, Australia

Full list of author information is available at the end of the article
}

stroke due to their impact on each other, or being directly associated with the risk of a second stroke [7-12]. Further to these health-risk behaviours, higher rates of depression and anxiety have been found among stroke survivors and have been identified as barriers to stroke survivors seeking counselling, advice, and education to modify health-risk behaviours post stroke [13]. Depression and anxiety have also both been proposed to have a significant negative association with HRQoL [14-16].

Providing behavioural interventions, such as brief advice, education, and counselling to modify patient health-risk behaviours, is evidence-based best practice

(C) The Author(s). 2019 Open Access This article is distributed under the terms of the Creative Commons Attribution 4.0 International License (http://creativecommons.org/licenses/by/4.0/), which permits unrestricted use, distribution, and 
according to clinical guidelines for stroke management [17]. However, audits have found that only about half of all patients receive behavioural interventions at the time of discharge [18]. There are a number of reasons for this evidence-practice gap, including practitioner lack of training, confidence, and skills and time to provide counselling and advice [19].

An online intervention may be one way to address these barriers while meeting the APEASE criteria for designing scalable interventions of Affordability, Practicality, Effectiveness, and cost-effectiveness, Acceptability, Safety, and Equity [20]. In Australia, more than $86 \%$ of households report Internet access at home [21]. Surveys show that up to $80 \%$ of patients have an interest in supplementing clinician-delivered support with Internet-delivered information, [22] and reviews have consistently demonstrated the effectiveness of online interventions among comorbid populations for reducing pain, disability, depression, and anxiety [23-25].

\section{Aims}

The primary aim of this study is to examine the effectiveness of an online healthy lifestyle intervention - Prevent 2nd Stroke (P2S) - at improving HRQoL among stroke survivors at 6 months' follow-up. A secondary aim is to examine the cost-effectiveness of the intervention compared to usual care. Additional secondary aims are to examine the effect of the online P2S programme on:

1. Four health behaviours (smoking, alcohol use, fruit and vegetable intake, and moderate physical activity)

2. Mental health (depression and anxiety levels)

3. Self-reported physical functioning and independent living, and

4. Hospital admission for recurrent stroke, myocardial infarction and all other causes

\section{Methods/design \\ Design}

A prospective, open, blinded end-point, randomised controlled trial (PeRSiST trial) with stroke survivors as the unit of randomisation will be conducted. Outcome assessments will be blinded. A flow chart of the trial design appears in Fig. 1. The protocol has been written following the Standard Protocol Items: Recommendations for Interventional Trials (SPIRIT) advice (see Additional file 1).

\section{Setting}

Participants will be recruited through two sources: (1) the Australian Stroke Clinical Registry (AuSCR) database, which is a national prospective opt-out enrolment registry for stroke and transient ischaemic attack (TIA) patients at 68 hospitals in six states of Australia, and (2) the Hunter Stroke Research Volunteer Register (HSRVR), this is a centralised database of people with stroke or TIA living in the Hunter region of the state of New South Wales who are willing to be contacted to participate in stroke research.

\section{Participants \\ Eligibility criteria}

Individuals will be included if they: are aged 18 years and over; have been admitted to an AuSCR hospital for a first episode of care for acute stroke or TIA (indexed stroke event) or are registered with the HSRVR; are between 6 and 36 months post stroke; are sufficiently fluent in English; and have sufficient facility in Internet use via a home device (e.g. computer or tablet device) or are willing to use public Internet services (e.g. public library).

\section{Exclusion criteria}

Individuals will not be eligible to participate in the study if they have documented evidence of a previous stroke (second to the indexed stroke event) or experience disability at a level that may limit their use of the programme (determined using a score of $\leq 3$ on the modified Rankin Scale) [26].

\section{Withdrawal}

If a participant wishes to withdraw consent, they will be able to at any point in time. They will be given the option to remove all their previously collected data or just remove consent for further data collection.

\section{Sample size}

A sample of 160 individuals will be needed per treatment arm at follow-up to enable the detection of a 0.25 standard deviation difference (a 6-point difference) in HRQoL at 6 months with $80 \%$ power and 5\% significance. This assumes a correlation between the baseline and follow-up HRQoL of 0.6, and a standard deviation of 24 points. Pilot data suggest that approximately $40 \%$ of participants recruited at baseline will complete follow-up at 6 months and, therefore, the research team aim to recruit 530 eligible consenting participants at baseline [27].

\section{Randomisation}

A random number generator embedded in the computer-assisted telephone interview (CATI) software will be used to allocate study participants to either receive the intervention or standard care after all baseline questions have been answered. Individuals will be randomised at a ratio of 1:1 in permuted blocks of randomly 
Initial mail out (AuSCR and HSRVR Teams)

(AuSCR: $n=2,700$; HSRVR: $n=250$ )

- In initial mail out, participants have to return consent and contact form

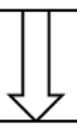

Enrolment Call (PeRSiST Team)

- Participants are screened for eligibility and contact details are confirmed

- The team arranges a time for participants to do the CATI baseline survey

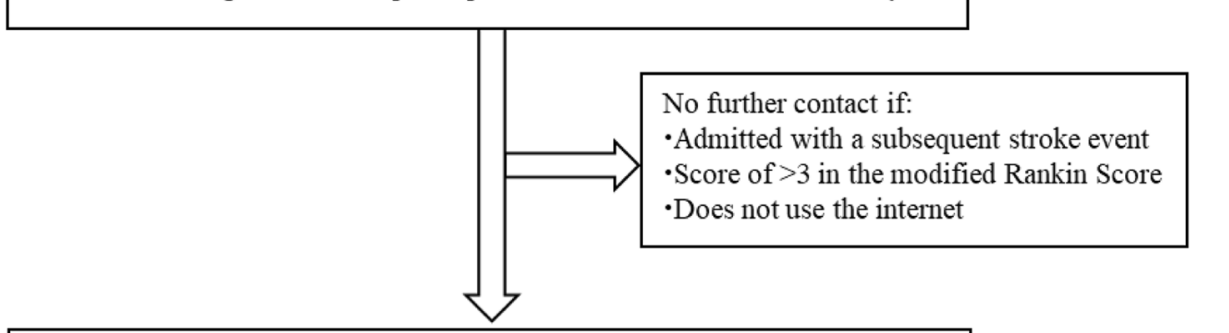

Baseline Survey (CATI Team)

$$
(\mathbf{n}=\mathbf{5 3 0})
$$

- Participants complete baseline survey

- After completion of the survey, participants are randomised into intervention and control groups

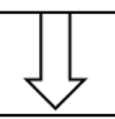

Intervention $(\mathbf{n}=\mathbf{2 6 5})$

- Participants receive access to the P2S program

- First contact is a letter/email detailing how to access the program

- Receive fortnightly texts/email reminders through intervention period ( 3 months), then receives monthly reminders until 6 month follow-up survey

- Intervention participants receive an expiration notification email (at 11 weeks) that their access to the P2S program at end of intervention (week 12)

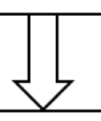

Six Month Survey (PeRSiST team \& CATI Team)

$$
\left(\mathbf{n}=\mathbf{3 2 0}^{*}\right)
$$

- Survival check conducted prior to sending survey list to CATI team

- CATI team contacts participants to complete six month survey

- Participants provided with reimbursement after survey completion by the P2S team

Collect MBS \& PBS data (PeRSiST Team)

Final data collection for health data collected by the research team after 6 month CATI completion

*Approximate value based on estimated loss to follow-uprates

Fig. 1 Schematic of the Prevent Second Stroke Trial (PeRSiST) study trial design 
varying size (e.g. four and six), stratified by state (New South Wales, Queensland, Western Australia, Victoria/ Tasmania), and type of stroke (ischaemic, haemorrhagic, TIA, other). The statistician will be blinded to the treatment group.

\section{Recruitment and screening}

AuSCR or HSRVR will screen individuals for eligibility criteria and will send out invitation packs on behalf of the trial research team to potentially eligible database registrants (i.e. individuals 6 to 36 months post first stoke, aged 18 years and older). Invitation packs will include a cover letter from AuSCR or HSRVR, a participant information statement describing the project Additional file 2 (Model participant information statement), and two consent forms (one for study participation Additional file 3 (Model participant consent form) and one to allow the researchers to access the individual's health care data (Medicare and Pharmaceutical Benefits Schemes; MBS and PBS) from the national registry). Participants will be asked to sign and return the consent forms in a replied, paid envelope to the research team. Due to potential physical limitations in the population being studied, carers will be able to consent on behalf of individuals by signing the consent form and indicating that they are a carer. Upon receipt of the signed consent forms, potential participants will be telephoned by the research team (enrolment call) to be screened regarding Internet and email access, and disability using the brief nine-item modified Rankin Scale. Potential participants will be, given an opportunity to ask questions about the study. Upon eligibility confirmation, participants will complete a baseline CATI after which they will be randomised.

Figure 1 outlines study recruitment and follow-up, while Fig. 2 contains an assessment schedule for the trial. To promote adherence to programme use and study follow-up, both groups will receive reminder texts (intervention fortnightly with additional email reminder; control monthly) during the intervention period of 12 weeks, then both groups will receive monthly reminder texts, including the approximate date for their next survey, until the 6-month follow-up. Intervention participants will have access to P2S for the 12 weeks. After 11 weeks, intervention participants will receive an email notifying them that their access to the P2S programme will expire within a week. Survival checks will be completed with AuSCR or HSRVR prior to telephone contact. All participants will be asked to complete a 6-month followup CATI. Participants will be reimbursed with an AU\$50 gift card after the completion of the 6-month survey.

\section{Intervention}

Participants randomised to receive the intervention will be sent an email and letter containing a link to P2S and their log-in details. The P2S programme is a modularised, tailored programme designed for individuals who have had a stroke to improve their HRQoL which may reduce their risk of a second stroke event. The P2S programme and its development is described in detail elsewhere [28]. Briefly, individuals are first presented with a loading module where they are asked questions to determine what information might be most relevant to the user. After completing the loading module, users are able to access the other seven modules (one module for each of the four health behaviours (Smoking, Alcohol Use, Diet, Physical Activity), one for Blood Pressure, one for Moods and Feeling, and the My Progress module (for participants to track their progress towards achieving goals).

During their 12-week access, intervention participants will be able to revisit sections of the programme as required, setting new goals, and recording progress throughout this time. Fortnightly text message and email reminders will be sent to prompt programme usage. Individuals will also receive a toll-free number that allows calls to the project team if they require assistance. Participants will be encouraged to use the online programme at least once a week and more often if they choose. Navigation through the programme will be flexible, with participants able to navigate around the programme freely, focussing on content areas of greatest interest or need.

\section{Minimal ethical care (control) group}

Participants randomised to the control group will receive usual stroke follow-up care and will be sent an email and letter with links to Internet addresses of readily available, generic online health programmes designed for the general population (The Australian Department of Health 'Quit Now', 'Australia's Physical Activity and Sedentary Behaviour Guidelines' and 'Eat for Health' sites; The National Health and Medical Research Council's 'Australian Guidelines to Reduce Health Risks from Drinking'; and the 'MOODGym' site). The use of an active control condition was chosen as it balances exposure to online health programmes and minimises the likelihood of changes in the treatment group due to non-specific therapeutic factors. P2S, which will only be accessible by entering user identification during the trial, will not be available to control group participants.

\section{Primary outcome measures}

The primary outcome measure will be HRQoL, measured using the EuroQol 5 Dimensions, 5 Levels (EQ5D-5 L) questionnaire [29], at 6 months. The EQ-5D-5 L 


\begin{tabular}{|c|c|c|c|c|c|}
\hline Contact / Visit & $\begin{array}{c}\mathbf{0} \\
\text { Mail out }\end{array}$ & $\begin{array}{c}1 \\
\begin{array}{c}\text { Enrolment } \\
\text { call }\end{array}\end{array}$ & $\begin{array}{c}2 \\
\text { Baseline } \\
\text { (CATI 1) }\end{array}$ & $\begin{array}{c}3 \\
\text { End of } \\
\text { intervention }\end{array}$ & $\begin{array}{c}4 \\
\text { Follow- } \\
\text { up 1 } \\
\text { (CATI 2) }\end{array}$ \\
\hline Week & & 0 & $1-2$ & 12 & 26 \\
\hline \multicolumn{6}{|l|}{ Treatment Period (12 weeks) } \\
\hline \multicolumn{6}{|l|}{ SCREENING, CONSENT \& Randomisation } \\
\hline Screening by AuSCR/HSRVR & $\mathrm{X}$ & & & & \\
\hline Informed Consent & $\mathrm{X}$ & & & & \\
\hline Receive participant contact details from & $\mathrm{X}$ & & & & \\
\hline Confirm enrolment to study (enrolment call) & & $\mathrm{X}$ & & & \\
\hline Randomisation (intervention receive program) & & & $\mathrm{X}$ & & \\
\hline Intervention expiration notification email & & & & $\mathrm{x}$ & \\
\hline \multicolumn{6}{|l|}{ MEASURES } \\
\hline \multicolumn{6}{|l|}{ Primary Objectives } \\
\hline Health related quality of life (EQ5D) & & & $\mathrm{x}$ & & $\mathrm{x}$ \\
\hline \multicolumn{6}{|l|}{ Secondary Objectives } \\
\hline Depression \& anxiety (PHQ-4) & & & $\mathrm{x}$ & & $\mathrm{x}$ \\
\hline Physical functioning and independent living & & & $\mathrm{X}$ & & $\mathrm{x}$ \\
\hline Smoking status & & & $\mathrm{x}$ & & $\mathrm{x}$ \\
\hline Diet quality (ARFS) & & & $\mathrm{x}$ & & $\mathrm{x}$ \\
\hline Alcohol (AUDIT-C) & & & $\mathrm{x}$ & & $\mathrm{x}$ \\
\hline Physical activity (GLTEQ) & & & $\mathrm{x}$ & & $\mathrm{x}$ \\
\hline Cost (CSIR) & & & $\mathrm{x}$ & & $\mathrm{x}$ \\
\hline Carer involvement questions & & & & & $\mathrm{x}$ \\
\hline Use of secondary prevention strategies & & & & & $\mathrm{x}$ \\
\hline \multicolumn{6}{|l|}{ Data from AuSCR/HSRVR } \\
\hline Age & $\mathrm{x}$ & & & & $\mathrm{x}$ \\
\hline Gender & $\mathrm{x}$ & & & & $\underline{x}$ \\
\hline Postcode & $x$ & & & & $\mathrm{x}$ \\
\hline ATSI & $\mathrm{x}$ & & & & $\underline{x}$ \\
\hline Country of birth & $\mathrm{x}$ & & & & $\mathrm{x}$ \\
\hline Hospital of admission & $\mathrm{x}$ & & & & $\mathrm{x}$ \\
\hline Type of stroke & $\mathrm{X}$ & & & & $\mathrm{x}$ \\
\hline Ability to walk independently on admission & $\mathrm{x}$ & & & & $\mathrm{x}$ \\
\hline Treated by stroke care unit (at hospital) & $\mathrm{X}$ & & & & $\mathrm{x}$ \\
\hline Survival check & & & & & $\mathrm{x}$ \\
\hline \multicolumn{6}{|l|}{ Medicare } \\
\hline Date of service & & & & & $\mathrm{x}$ \\
\hline Medicare item number and description & & & & & $\mathrm{x}$ \\
\hline Provider charge & & & & & $\mathrm{x}$ \\
\hline Schedule fee & & & & & $\mathrm{x}$ \\
\hline Benefit paid & & & & & $\mathrm{x}$ \\
\hline Patient out of pocket & & & & & $\mathrm{x}$ \\
\hline Bill type & & & & & $\mathrm{x}$ \\
\hline Scrambled ordering provider number & & & & & $\mathrm{x}$ \\
\hline Scrambled rendering provider number & & & & & $\mathrm{x}$ \\
\hline Date of referral & & & & & $\mathrm{x}$ \\
\hline Rendering provider postcode & & & & & $\mathrm{x}$ \\
\hline Ordering provider postcode & & & & & $\mathrm{x}$ \\
\hline Hospital indicator & & & & & $\mathrm{x}$ \\
\hline \multicolumn{6}{|l|}{ Pharmaceutical Benefits Scheme (PBS) } \\
\hline Date of supply & & & & & $\mathrm{x}$ \\
\hline Date of prescribing & & & & & $\mathrm{x}$ \\
\hline PBS item code & & & & & $\mathrm{x}$ \\
\hline Item description & & & & & $\mathrm{x}$ \\
\hline Patient category & & & & & $\mathrm{x}$ \\
\hline Patient contribution & & & & & $\mathrm{x}$ \\
\hline Net benefit & & & & & $\mathrm{x}$ \\
\hline Scrambled prescriber number & & & & & $\mathrm{x}$ \\
\hline Pharmacy postcode & & & & & $\mathrm{X}$ \\
\hline Form category & & & & & $\mathrm{x}$ \\
\hline ATC code & & & & & $\mathrm{x}$ \\
\hline ATC name & & & & & $\mathrm{x}$ \\
\hline \multicolumn{6}{|l|}{ Adherence to intervention (QuON) } \\
\hline Timing data & & & & & $\mathrm{x}$ \\
\hline Usage data & & & & & $\mathrm{X}$ \\
\hline
\end{tabular}

Fig. 2 Assessment schedule of enrolment, interventions, and assessments for participants 
consists of two stages of questions. Stage 1 contains five questions in the domains of mobility, self-care, usual activities, pain/discomfort, and mood (the EQ-5D). Each dimension has five levels: no problems, slight problems, moderate problems, severe problems, and extreme problems. The second stage adapts the EuroQol Visual Analogue Scale (EQ VAS), and asks respondents to numerically rate global health status from 0 to 100 , and can be administered verbally [30, 31]. The justification for the EQ-5D-5 L is that it: (1) is very short and simple, taking 2-3 min and can be administered as a telephone interview; (2) is responsive to change in patients who have had a stroke; (3) is composed of multi-item scales and single items to examine differential effects; (4) has been used in, and is recommended for, stroke trials [32]; and (5) the construct, concurrent and discriminant validity, reliability, and acceptability (based on response rates and missing data) of the instrument with stroke patients has been well established [33].

\section{Secondary outcome measures Cost-effectiveness}

The main reportable outcomes for the cost-effectiveness analysis of the intervention will be (1) an average cost-effectiveness ratio, and (2) an incremental cost-effectiveness ratio, per Quality-adjusted Life Year (QALY). A modified version of the Client Service Receipt Inventory (CSRI) will be used in conjunction with the MBS and PBS data to determine use of health services (frequency, length and cost of physician consultations; allied health care; inpatient stays; outpatient episodes; medication use) and potentially other economic impacts (such as time off work due to disability) [34].

\section{Smoking status}

At baseline, a standard and previously validated item will assess smoking status 'Do you currently smoke any tobacco products?' (Daily, At least once a week, Less often than once a week, Not at all) [35]. At follow-up, 7-day point-prevalence abstinence (PPA) will be used to assess quit success using recommended items to determine the proportion of participants who have not smoked any tobacco in the preceding 7 days 'Have you smoked at least part of a cigarette in the last 7 days?' Seven-day PPA has high concurrent validity and reliability [36].

\section{Alcohol consumption}

The brief Alcohol Use Disorders Identification Test Consumption (AUDIT-C), [37] will be used. The AUDIT-C is a three-item version of the more extensive AUDIT and has been found to be a valid and reliable screening tool for heavy drinking and alcohol abuse or dependence. Scoring for the AUDIT-C ranges from 0 to 12 with cut-offs of 3 for women (sensitivity 66-73\%, specificity 91-94) [38, 39] and 4 for men are used to indicate heavy drinking (sensitivity $86 \%$, specificity $72-89 \%$ ) [39].

\section{Fruit and vegetable intake}

The Australian Recommended Food Score (ARFS) [40] will be used to assess fruit and vegetable consumption, a measure which has been validated in adults. The ARFS is strongly correlated with food frequency questionnaire nutrient intakes (fibre, vitamin A, beta-carotene, vitamin $\mathrm{C}$, and minerals). To determine salt intake, participants will be asked 'How often do you add salt to your cooking?' and 'How often do you use salt at the table?' (Never/rarely, Sometimes, Usually, Always) [41].

\section{Physical activity}

Physical activity will be measured using the Godin Leisure Time Exercise Questionnaire (GLTEQ), [42] which measures the number of occurrences in a week in which a participant engages in periods of mild, moderate or vigorous exercise for $10 \mathrm{~min}$ or longer. Weights are then applied to the number of weekly occurrences of each type of exercise (mild, moderate, vigorous) and summed to calculate the number of metabolic equivalent or MET-minutes of exercise per week; it has acceptable reliability and validity.

\section{Depression and anxiety}

Depression and anxiety levels will be assessed using The Patient Health Questionnaire four-item (PHQ-4) [43]. The PHQ-4 is a validated and ultra-brief screening measure for both anxiety and depression. Scores range from 0 to 12, with categories of psychological distress being: none (0-2); mild (3-5); moderate (6-8); and severe (9-12). Subscale categories for anxiety and depression both range from 0 to 6 with a score of 3 or more considered positive.

\section{Physical functioning and independent living}

Physical functioning and independent living will be measured using the Barthel Index (BI) [44, 45] and Instrumental Activities of Daily Living (IADL) scales [46]. The BI measures activities of daily life on a 10-point scale related to self-care and mobility. The IADL questions attempt to capture the ability to live independently in the community.

\section{Other secondary outcome measures}

Other measures include hospital admission for recurrent stroke, myocardial infarction and all other causes, and data from medical health records. These data will be collected from national health care databases and will be used to inform resource-use assumptions in the cost-effectiveness analysis for 
patients utilising hospitals in health districts without access to an equivalent intervention.

\section{Additional measures}

Patient and disease characteristics including age, gender, postcode, indigenous status, country of birth, hospital, type of stroke, whether the patient was able to walk independently on admission, and whether the patient was treated in a stroke care unit will also be collected. Adherence to the intervention, carer involvement, blood pressure awareness, and use of secondary prevention strategies will also be collected at 6 months. Programme usage data (i.e. timing and usage) will be collected as part of backend analytics from the web-platform hosting the $\mathrm{P} 2 \mathrm{~S}$ programme.

\section{Blinding}

All baseline and follow-up telephone assessments will be conducted by independent health survey contractors blinded to experimental condition. Due to the nature of the interventions, blinding participant allocation and participants will be notified of the condition to which they have been assigned.

\section{Data collection}

A commercial research company will be commissioned to collect baseline and follow-up data by CATI survey. MBS and PBS health data will be extracted from the national database at baseline and 6 months with the assistance of Department of Human Services, Canberra.

Study participant information and consent forms will have a unique study identification (ID) number. After formal enrolment into the trial participants will be assigned that unique study ID, allowing data to be stored in a re-identifiable format. The ID number will be used to identify participant data sets for comparison (i.e. baseline and follow-up surveys). Participant identifying details (i.e. names and contact details collected to enable follow-up) will be stored separate to other data, while the ID number will be attached to both.

\section{Statistical analysis \\ Primary analysis}

Will be conducted according to the intention-to-treat (ITT) principle, comparing the change in HRQoL between groups from baseline to 6-month follow-up. The comparison will be done using a linear regression model, where the outcome variable will be the EQ-5D-5L HRQoL score, and the main predictors of interest will be the baseline value of the outcome variable, and treatment group. The two stratifying variables, state of residence within Australia and stroke type at baseline, will be included in the model as covariates. The primary method of dealing with missing data will be through multiple imputation, this will provide a sensitivity analysis exploring how robust the results are when considering missing values. The missing data will be explored to test the underlying assumptions of multiple imputations. Adjusting for potentially imbalances in important prognostic variables and pattern mixture models will be used to correct for violations in the missing at random assumption.

\section{Secondary analysis}

Generalised linear mixed models will be used to examine between-group differences in changes in secondary outcomes between baseline and follow-up; appropriate distributional families and link functions will be used according to outcome type. The models will include fixed effects for time, group and the interaction between time and group, as well as stratification variables. Random subject-level intercepts will be included to account for serial correlations. Associations between programme usage and outcome will be examined where greater adherence to the programme will be expected to be associated with improved outcomes.

\section{Cost-effectiveness analysis}

In this study, a cost-utility analysis will be undertaken from the perspective of health care providers to identify whether the resources consumed to achieve the outcomes represent a cost-effective improvement over usual stroke follow-up care. The analysis will adhere to guidelines for conducting and reporting health economic evaluations [47, 48]. Given that the majority of resource savings are anticipated to arise following conclusion of the intervention, a patient lifetime time-horizon will be modelled founded on the trial outcomes. The primary measure of effectiveness for the economic evaluation will comprise HRQoL, as measured by EQ-5D-5 L, from which QALYs will be calculated using Australian utility weights. Resource utilisation for the respective trial arms will include: intervention development costs, health care costs derived from the AuSCR hospital International Classification of Disease (ICD) data, the CSRI data, and the MBS/ PBS data. The $I C D$ data will be translated to an estimate for cost differences between arms using allocations to diagnostic-related groups and according cost weights. It is implicitly assumed that differences between trial arms for non-stroke utilisation of inpatient services will be random. This assumption will be tested with the CSRI inpatient data and admitted patient data from a subset of health districts. Analysis of the QALYs and resource-use estimates will derive average and incremental cost-effectiveness 
ratios. Appropriate sensitivity and uncertainty analysis will be conducted.

A full statistical analysis plan will be written prior to analysis.

\section{Data monitoring}

A data monitoring committee is not required for observational studies. In lieu of this committee, the research team will meet weekly to discuss the day-to-day running of the project and the full investigator team will be contacted monthly. This team will discuss any broader concerns arising from the research that cannot be managed by the research team, and will monitor the ethics and safety of the trial and will convene as required throughout the duration of the trial. If the research team is made aware of any negative events that may be the result of study participation, the research and investigator teams will be responsible for discussing the event, and any participation or protocol changes which may result, at their weekly/monthly meetings.

\section{Discussion}

To date, there are limited findings on the impact of online behaviour-change interventions for improvements in HRQoL and the prevention of second stroke among stroke survivors. Determining if there is the potential to increase care provision through online platforms may help to bring care provision in line with current recommendations, or go further, and increase the quality of life of users and prevent further ill-health. The results of this randomised controlled trial should provide further evidence regarding online interventions and their ability to improve HRQoL and support stroke survivors.

\section{Trial status}

Protocol version number and date: Version 3, dated 1 September 2018.

Recruitment start date: 1 March 2018.

Expected recruited completion date: 28 February 2019.

\section{Additional files}

Additional file 1: Standard Protocol Items: Recommendations for Interventional Trials (SPIRIT) Checklist. (PDF 172 kb)

Additional file 2: Model participant information statement. Example information statement provided to potential participants about the trial. (PDF $177 \mathrm{~kb}$ )

Additional file 3: Model participant consent form. Example consent form provided to potential participants to enrol in the trial. (PDF $222 \mathrm{~kb}$ )

\section{Abbreviations}

APEASE : Affordability, Practicality, Effectiveness, and cost-effectiveness, Acceptability, Safety, and Equity; AuSCR: Australian Stroke Clinical Registry; CATI: Computer-assisted telephone interview; CSRI: Client Service Receipt Inventory; HRQoL: Health-related quality of life; HSRVR: Hunter Stroke Research Volunteer Register; ICD: International Classification of Disease;
ID: Identification; ITT: Intention-to-treat; MBS : Medicare Benefits Scheme; P2S: Prevent 2nd Stroke; PBS: Pharmaceutical Benefit Scheme; PeRSiST : Prevent Second Stroke Trial; QALY: Quality-adjusted Life Year; TIA : Transient ischaemic attack

\section{Acknowledgements}

Not applicable.

\section{Sponsors}

The study sponsor is the University of Newcastle, University Drive, Callaghan, NSW 2308.

The study sponsor and funder have had no input into the study design; collection, management, analysis and interpretation of data; writing of the report; or the decision to submit the report for publication and will not have any such input in the future.

\section{Authors' contributions}

BB conceived the study; participated in its design, coordination, and implementation; and helped draft the manuscript. AG participated in trial development, coordination and implementation and drafted the manuscript $N S, M P, A B, P M, A T, C C, R C$, and $C L$ participated in the design of the study, contributed to trial development, and advised on implementation. CO participated in design of the study and advised on statistical analysis. AS and SD participated in design of the study and advised on the cost-effectiveness analysis. SM, OW, AMJD, and BC participated in trial development and implementation. All authors read and approved the final manuscript.

\section{Funding}

This study was funded by a grant from the National Health and Medical Research Council (NHMRC) (APP1125429). AG is supported by a post-doctoral fellowship from the Heart Foundation. AB is supported by a NHMRC research fellowship. AMJD is supported by a Research Training Program (RTP), University of Newcastle PhD Scholarship and Hunter Medical Research Institute/ Emlyn and Jennie Thomas Postgraduate Medical Research Scholarship. The funder of the study will have no role in study design, data collection, data analysis, data interpretation or writing of the report. The corresponding author will have full access to all the data in the study and had final responsibility for the decision to submit for publication.

\section{Availability of data and materials}

Not applicable.

Ethics approval and consent to participate

This study has received ethics approval from the University of Newcastle Human Research Ethics Committee (H-2017-0051). Informed consent will be obtained from all study participants.

Consent for publication

Not applicable.

\section{Competing interests}

The authors declare that they have no competing interests.

\section{Author details}

${ }^{1}$ School of Medicine and Public Health, Faculty of Health and Medicine, The University of Newcastle, 1 University Drive, Callaghan, NSW 2308, Australia.

${ }^{2}$ The University of Newcastle, School of Biomedical Sciences and Pharmacy, Faculty of Health and Medicine, 1 University Drive, Callaghan, NSW 2308, Australia. ${ }^{3}$ Hunter Medical Research Institute (HMRI), New Lambton Heights, NSW 2305, Australia. ${ }^{4}$ Hunter New England Local Health District, John Hunter Hospital, New Lambton Heights, NSW 2305, Australia. ${ }^{5}$ IMPACT Strategic Research Centre, School of Medicine, Barwon Health, Deakin University, PO Box 291, Geelong, VIC, Australia. 'Department of Psychiatry, Level 1 North, Main Block, Royal Melbourne Hospital, University of Melbourne, Parkville, VIC, Australia. ${ }^{7}$ School of Health Sciences, Faculty of Health and Medicine, The University of Newcastle, 1 University Drive, Callaghan, NSW 2308, Australia. 


\section{Received: 28 February 2019 Accepted: 19 July 2019}

\section{Published online: 09 August 2019}

\section{References}

1. O'Donnell MJ, Chin SL, Rangarajan S, Xavier D, Liu L, Zhang H, et al. Global and regional effects of potentially modifiable risk factors associated with acute stroke in 32 countries (INTERSTROKE): a casecontrol study. Lancet. 2016;388(10046):761-75.

2. Bardage C, Isacson DGL. Hypertension and health-related quality of life: an epidemiological study in Sweden. J Clin Epidemiol. 2001;54(2):172-81.

3. Jeon NE, Kwon KM, Kim YH, Lee JS. The factors associated with health-related quality of life in stroke survivors age 40 and older. Ann Rehabil Med. 2017; 41(5):743-52.

4. Gordon CD, Wilks R, McCaw-Binns A. Effect of aerobic exercise (walking) training on functional status and health-related quality of life in chronic stroke survivors. A randomized controlled trial. Stroke. 2013;44(4):1179-81.

5. Brown DR, Carroll DD, Workman LM, Carlson SA, Brown DW. Physical activity and health-related quality of life: US adults with and without limitations. Qual Life Res. 2014;23(10):2673-80.

6. Smedt DD, Clays E, Annemans L, Boudrez H, Sutter JD, Doyle F, et al. The association between self-reported lifestyle changes and health-related quality of life in coronary patients: the EUROASPIRE III survey. Eur J Prev Cardiol. 2014;21 (7):796-805.

7. Stroke Foundation. High blood pressure and stroke 2017. Available from: https://strokefoundation.org.au/en/About-Stroke/Prevent-Stroke/High-bloodpressure.

8. Mazzaglia G, Britton AR, Altmann DR, Chenet L. Exploring the relationship between alcohol consumption and non-fatal or fatal stroke: a systematic review. Addiction. 2001;96(12):1743-56.

9. Shah RS, Cole JW. Smoking and stroke: the more you smoke the more you stroke. Expert Rev Cardiovasc Ther. 2010;8(7):917-32.

10. Epstein KA, Viscoli CM, Spence JD, Young LH, Inzucchi SE, Gorman M, et al. Smoking cessation and outcome after ischemic stroke or TIA. Neurology. 2017;89(16):1723-9.

11. Kyu HH, Bachman VF, Alexander LT, Mumford JE, Afshin A, Estep K, et al. Physical activity and risk of breast cancer, colon cancer, diabetes, ischemic heart disease, and ischemic stroke events: systematic review and dose-response meta-analysis for the Global Burden of Disease Study 2013. bmj. 2016;354:i3857.

12. Hu D, Huang J, Wang Y, Zhang D, Qu Y. Fruits and vegetables consumption and risk of stroke. Stroke. 2014;45(6):1613-9.

13. Allison R, Evans PH, Kilbride C, Campbell JL. Secondary prevention of stroke: using the experiences of patients and carers to inform the development of an educational resource. Fam Pract. 2008;25(5):355-61.

14. Tang WK, Lau CG, Mok V, Ungvari GS, Wong KS. Impact of anxiety on health-related quality of life after stroke: a cross-sectional study. Arch Phys Med Rehabil. 2013;94(12):2535-41.

15. Hsu N-W, Tsao H-M, Chen H-C, Chou P. Anxiety and depression mediate the health-related quality of life differently in patients with cardiovascular disease and stroke-preliminary report of the Yilan Study: a populationbased community health survey. PLoS One. 2014;9(9):e107609.

16. Godwin KM, Ostwald SK, Cron SG, Wasserman J. Long-term health related quality of life of survivors of stroke and their spousal caregivers. J Neurosci Nurs. 2013;45(3):147-54.

17. Stroke Foundation. (Australian) Clinical guidelines for stroke management 2017. Melbourne: Stroke Foundation; 2017.

18. National Stroke Foundation. National Stroke Audit: Acute Services Report 2015. 2015.

19. Wolfe CDA, Redfern J, Rudd AG, Grieve AP, Heuschmann PU, McKevitt C. Cluster randomized controlled trial of a patient and general practitioner intervention to improve the management of multiple risk factors after stroke. Stroke. 2010;41(11):2470-6.

20. Michie $S$, van Stralen MM, West R. The behaviour change wheel: a new method for characterising and designing behaviour change interventions. Implement Sci. 2011;6(1):42.

21. Australian Bureau of Statistics. Household use of information technology, Australia, 2014-15. 2016.

22. Monnier J, Laken M, Carter CL. Patient and caregiver interest in Internetbased cancer services. Cancer Pract. 2002;10(6):305-10.

23. Eccleston C, Fisher E, Craig L, Duggan GB, Rosser BA, Keogh E. Psychological therapies (Internet-delivered) for the management of chronic pain in adults. Cochrane Database Syst Rev. 2014;2. Article \# CDO10152.
24. Buhrman M, Syk M, Burvall O, Hartig T, Gordh T, Andersson G. Individualized guided Internet-delivered cognitive-behavior therapy for chronic pain patients with comorbid depression and anxiety: a randomized controlled trial. Clin J Pain. 2015;31(6):504-16.

25. Andersson G, Hedman E. Effectiveness of guided internet-based cognitive behavior therapy in regular clinical settings. Verhaltenstherapie. 2013;23(3): 140-8.

26. Quinn TJ, Lees KR, Hardemark H-G, Dawson J, Walters MR. Initial experience of a digital training resource for modified Rankin scale assessment in clinical trials. Stroke. 2007;38(8):2257-61.

27. Denham AMJ, Halpin S, Twyman L, Guillaumier A, Bonevski B. Prevent 2nd Stroke: a pilot study of an online secondary prevention program for stroke survivors. Aust N Z J Public Health. 2018:42(5):484-90.

28. Denham, AMJ, Guillaumier A, McCrabb S, Turner A, Baker A, Spratt N, et al. "Development of an online secondary prevention programme for stroke survivors: Prevent 2nd Stroke." BMJ Innovations. bmjinnov-2017000257.

29. Group TE. EuroQol-a new facility for the measurement of health-related quality of life. Health Policy. 1990;16(3):199-208.

30. Chatterji R, Naylor JM, Harris IA, Armstrong E, Davidson E, Ekmejian R, et al. An equivalence study: are patient-completed and telephone interview equivalent modes of administration for the EuroQol survey? Health Qual Life Outcomes. 2017;15(1):18.

31. McPhail S, Lane P, Russell T, Brauer SG, Urry S, Jasiewicz J, et al. Telephone reliability of the Frenchay Activity Index and EQ-5D amongst older adults. Health Qual Life Outcomes. 2009;7(1):48.

32. Gibbons EJ, Fitzpatrick R, Jenkinson C. A structured review of Patientreported Outcome Measures (PROMs) for stroke. Oxford: University of Oxford; 2009.

33. Golicki D, Niewada M, Buczek J, Karlińska A, Kobayashi A, Janssen M, et al. Validity of EQ-5D-5L in stroke. Qual Life Res. 2015;24(4):845-50.

34. Patel A, Rendu A, Moran P, Leese M, Mann A, Knapp M. A comparison of two methods of collecting economic data in primary care. Fam Pract. 2005; 22(3):323-7.

35. Mullins R, Borland R. Changing the way smoking is measured among Australian adults: a preliminary investigation of Victorian data. Quit Eval Stud. 1998;9:163-73.

36. Hughes JR, Keely JP, Niaura RS, Ossip-Klein DJ, Richmond RL, Swan GE. Measures of abstinence in clinical trials: issues and recommendations. Nicotine Tob Res. 2003;5(1):13-25.

37. Bush K, Kivlahan DR, McDonell MB, Fihn SD, Bradley KA. The AUDIT alcohol consumption questions (AUDIT-C): an effective brief screening test for problem drinking. Arch Intern Med. 1998;158(16):1789-95.

38. Bradley KA, Bush KR, Epler AJ, Dobie DJ, Davis TM, Sporleder JL, et al. Two brief alcohol-screening tests from the Alcohol Use Disorders Identification Test (AUDIT): validation in a female Veterans Affairs patient population. Arch Intern Med. 2003;163(7):821-9.

39. Bradley KA, DeBenedetti AF, Volk RJ, Williams EC, Frank D, Kivlahan DR. AUDIT-C as a brief screen for alcohol misuse in primary care. Alcohol Clin Exp Res. 2007;31(7):1208-17.

40. Collins CE, Burrows TL, Rollo ME, Boggess MM, Watson JF, Guest M, et al. The comparative validity and reproducibility of a diet quality index for adults: the Australian Recommended Food Score. Nutrients. 2015: 7(2):785-98.

41. Sarmugam R, Worsley A, Wang W. An examination of the mediating role of salt knowledge and beliefs on the relationship between socio-demographic factors and discretionary salt use: a cross-sectional study. Int J Behav Nutr Phys Act. 2013;10(1):25.

42. Eisenmann J, Milburn N, Jacobsen L, Moore S. Reliability and convergent validity of the Godin Leisure-Time Exercise Questionnaire in rural 5th-grade school-children. J Hum Mov Stud. 2002;43(2):135-49.

43. Kroenke K, Spitzer RL, Williams JB, Löwe B. An ultra-brief screening scale for anxiety and depression: the PHQ-4. Psychosomatics. 2009;50(6):613-21.

44. Mahoney Fl, Barthel D. Functional evaluation: The Barthel Index. Md State Med J. 1965;14:56-61.

45. Middleton S, Levi C, Ward J, Grimshaw J, Griffiths R, D'Este C, et al. Fever, hyperglycaemia and swallowing dysfunction management in acute stroke: a cluster randomised controlled trial of knowledge transfer. Implement Sci. 2009; $4(1): 16$

46. Katz S. Assessing self-maintenance: activities of daily living, mobility, and instrumental activities of daily living. J Am Geriatr Soc. 1983;31(12):721-7. 
47. Drummond MF, Sculpher MJ, Claxton K, Stoddart GL, Torrance GW. Methods for the economic evaluation of health care programmes. Oxford: Oxford university press; 2015.

48. Husereau D, Drummond M, Petrou S, Carswell C, Moher D, Greenberg D, et al. Consolidated Health Economic Evaluation Reporting Standards (CHEERS) Statement. Eur J Health Econ. 2013;14(3):367-72.

\section{Publisher's Note}

Springer Nature remains neutral with regard to jurisdictional claims in published maps and institutional affiliations.

Ready to submit your research? Choose BMC and benefit from:

- fast, convenient online submission

- thorough peer review by experienced researchers in your field

- rapid publication on acceptance

- support for research data, including large and complex data types

- gold Open Access which fosters wider collaboration and increased citations

- maximum visibility for your research: over $100 \mathrm{M}$ website views per year

At $\mathrm{BMC}$, research is always in progress.

Learn more biomedcentral.com/submissions 